\title{
Manfred Bierwisch
}

\section{Die Illusion einer »Stunde Null«}

\author{
Wissenschaft und Wiedervereinigung
}

Der völlige Zusammenbruch der Verwaltung einer Gesellschaft, ihrer Bildungs- und Forschungseinrichtungen kann vielfältige Reaktionen auslösen, von Abwehr, Furcht und Bedenken bis zu Erwartungen, Plänen und Hoffnungen, je nach der Lage des Betrachters oder Betroffenen. In diesem Sinn hat der Fall der Berliner Mauer alle möglichen Erwartungen und Intentionen geweckt und eine Fülle bemerkenswerter Aktivitäten ausgelöst. Die Lage nach dem Ende des Kalten Kriegs in Ostdeutschland war dennoch einzigartig im Vergleich zu anderen osteuropäischen Ländern, denn die Integration dessen, was der sogenannte »real existierende Sozialismus « hervorgebracht hatte, in eine hochentwickelte bürgerliche Gesellschaft hatte nirgends eine Parallele. Ob zum Vorteil oder zum Nachteil, keines der Länder des vormaligen sozialistischen Blocks musste sich mit einem westlichen Teil vereinigen. Diese Vereinigung hatte einschneidende Konsequenzen auch für die akademische Gemeinschaft und ihre Organisationen, nicht allein, aber vornehmlich für die östliche Seite.

Aus verschiedenen Gründen gab es viele Brüche, aber es war keine »Stunde Null «, es war kein Neuanfang. Die Veränderungen und Umbrüche bedeuteten zwar in vielerlei Hinsicht einen neuen Start, aber sie ereigneten sich nicht in einem leeren Raum, sie beinhalteten keine Möglichkeiten, etwas wie auf einem unbeschriebenen Blatt zu entfalten. Sie waren stattdessen abhängig von den Bedingungen auf beiden Seiten des geteilten Deutschlands, die sich aus der jeweiligen Geschichte der beiden Landesteile ergeben hatten.

\section{Vorgeschichte der Vereinigung}

\subsection{Das Ende des Zweiten Weltkriegs}

Ausgehend von der gemeinsam verursachten Katastrophe, hatten die beiden Teile Deutschlands eine dramatisch auseinandergehende Nachkriegsentwicklung. In der Ostzone, die von der Sowjetunion kontrolliert wurde, wurde eine antifaschistische Orientierung proklamiert. Durch diese Entscheidung verschwand zwar die ideologische Kontaminierung der Bevölkerung nicht, aber die öffentliche Ausrichtung und die offizielle Politik waren strikt antifaschistisch.

Diese anfängliche Verpflichtung manifestierte sich auf verschiedene Weise, nicht zuletzt in der Rückkehr bedeutender Emigranten, die Deutschland in der Zeit des Nationalsozialismus hatten verlassen müssen und jetzt in Ostdeutschland die Chance für ein besseres Deutschland sahen. Das war für die Universitäten im All- 
gemeinen wichtig, aber im Besonderen auch für die neuen Perspektiven in den Geistes- und Sozialwissenschaften.

Die sowjetische Herrschaft in der Zeit des Stalinismus bedeutete jedoch einen wachsenden Dogmatismus und eine ideologische Indoktrination in allen Feldern und hatte zur Folge, dass die kommunistische Partei in allen Personalangelegenheiten und Fragen von substanzieller Bedeutung das letzte Wort hatte. Eine Folge davon war, dass viele der Rückkehrer den sozialistischen Teil Deutschlands wieder verließen, von denen Ernst Bloch, Hans Mayer und Alfred Kantorowicz zu den spektakuläreren Fällen gehörten; zahlreiche Forschungsprojekte wurden aus ideologischen Gründen behindert, viele Leute wurden von der Lehre abgehalten, und es gab alle Arten von Problemen mit der Publikation von Texten. Schwere Einbußen in Lehre und Forschung waren unvermeidbar. Man muss allerdings auch hinzufügen, dass trotz all dieser dogmatischen und inkompetenten Einmischungen manche respektablen Ergebnisse erreicht wurden - nicht nur in den Naturwissenschaften. So wurde etwa das erste umfassende wissenschaftliche Wörterbuch der modernen deutschen Sprache in der Akademie der Wissenschaften der DDR produziert - mit einigen Defiziten, die aus ideologisch motivierten Eingriffen stammten, die aber im Nachhinein leicht zu beheben waren.

Eine völlig andere Entwicklung fand in den drei Westzonen Nachkriegsdeutschlands statt, die sich 1949 zur Bundesrepublik Deutschland vereinigten. Rigide konservative öffentliche Meinungen und Orientierungen bestimmten deren Anfangsphase, in der nicht nur die fatalen Erinnerungen an den Zweiten Weltkrieg verdrängt wurden, sondern auch öffentliche Ämter von Personen wahrgenommen wurden, die diese bereits in der Periode des Nationalsozialismus bekleidet hatten. Erst die Studentenbewegung der späten 1960er Jahre brach diese verschwiegene Kontinuität auf. Andererseits garantierte aber die Politik und die Orientierung, die von den Alliierten gefördert worden war, die Standards der liberalen Gesellschaft schon von Beginn an und erlaubte der akademischen Gemeinschaft, zu einem produktiven und tatsächlich recht erfolgreichen Teil der internationalen wissenschaftlichen Gemeinschaft zu werden. Autonome Einrichtungen der akademischen Selbstverwaltung hatten sich entwickelt, insbesondere die Deutsche Forschungsgemeinschaft (DFG), die Forschungsprojekte unterstützte und bewertete, die dafür sorgte, dass alternative Theorien und Methoden verfolgt werden konnten, dass zu kontroversen Debatten ermutigt wurde, kurz, dass der ganze Katalog von normaler Erziehung, Studium und Forschung zur Verfügung stand - ein erstaunliches Ergebnis, das nicht erwartet werden konnte, als die Kriegsverbrechen in einer hoffnungslosen Situation geendet hatten.

\subsection{Die letzte Phase der DDR und ibrer akademischen Institutionen}

Obwohl niemand den Fall der Berliner Mauer 1989 vorhergesehen hatte, waren die wachsenden Defizite des sogenannten »real existierenden Sozialismus « in Ostdeutschland insbesondere in Bezug auf die wirtschaftliche Situation für jeden Beobachter offensichtlich. Der tatsächliche Umbruch kam dann auch nicht wirklich als Überraschung. Als Gorbatschow in der Sowjetunion mit dem begann, was er peres- 
troika nannte, musste jedem klar sein, dass die Dinge nicht unverändert bleiben würden. Die Lage spitzte sich zu mit den verbotenen, aber stetig anwachsenden "Montagsdemonstrationen « in Leipzig, die ein völlig unvorhergesehenes Ereignis darstellten, das den Vorsitzenden der Regierungspartei dazu zwang, zurückzutreten. Das war ein bis dahin undenkbarer Schritt, der zu einer Reihe von weiteren Turbulenzen führte, darunter auch zur unkontrollierten Öffnung der Grenze, was dann schon fast zwangsläufig die Selbstauflösung der Regierung zur Folge hatte.

Initiiert durch diese dramatischen Veränderungen, begannen in der letzten Phase der DDR viele Aktivitäten in praktisch allen Einrichtungen, Betrieben und Verwaltungen - Versuche, Dinge zu modifizieren, zu korrigieren und anzupassen, bei denen man eine Korrektur für nötig befand. In besonderer Weise aktiv waren in dieser Hinsicht die Universitäten und Fakultäten, die Akademie der Wissenschaften, ihre Einrichtungen und ähnliche Forschungseinheiten. Mehrere interne Maßnahmen wurden ergriffen, um unangemessene Entscheidungen aufzuheben, unkorrekt behandelte Mitarbeiter zu rehabilitieren, die Situation durch Aufträge in letzter Minute zu verbessern. Viele Einrichtungen ersetzten diskreditierte Direktoren durch demokratisch gewählte Nachfolger, alle Universitäten ernannten neue Rektoren. In vielen Stellen und Organisationen wurden gewählte Repräsentanten eingeführt, um für die weitere Entwicklung gerüstet zu sein, soweit dafür überhaupt Möglichkeiten erkennbar waren. Mit anderen Worten: Eine Vielzahl von Aktivitäten - einschließlich harscher Kontroversen - manifestierte die Wiederentdeckung der Selbstbestimmung in der akademischen Gemeinschaft.

All dies aber endete und wurde obsolet, als von der Volkskammer, dem letzten und einzigen frei gewählten Parlament der DDR, die politische Einigung beschlossen wurde. Dazu muss auch gesagt werden, dass in der letzten Phase der DDR Mitglieder des vormaligen Establishments und Repräsentanten des alten politischen Systems ihr Erbe zu retten oder zumindest die Spuren mancher Dinge zu verwischen versuchten, für die sie verantwortlich gewesen waren. Das galt insbesondere für die örtlichen Behörden der Staatssicherheit, die sehr effizient viele Dokumente vernichteten, um sie unzugänglich zu machen für die Begründungen späterer Entscheidungen und Anordnungen.

\section{Vereinigung}

\subsection{Die Rabmenbedingungen der Vereinigung: Massive Asymmetrien}

Es ist weithin bekannt und wird doch häufig ignoriert, dass die Vereinigung von Ost- und Westdeutschland ein extrem asymmetrischer und aus nahezu unvermeidbaren Gründen auch ein oft völlig einseitiger Prozess war. Er trug sich zwischen zwei Systemen zu, die in fast jeder Hinsicht unterschiedlich waren. Die offensichtlichste Differenz war die Größe: Die Proportion der zwei Teile in Bezug auf räumliche Größe und Bevölkerungszahl war 4:1, und der Unterschied war noch größer bei der Wirtschaftskraft - zum Glück, könnte man sagen, im Blick auf die folgenden Asymmetrien. 
In inhaltlichen Fragen verhielten sich die Dinge komplizierter. Die Bundesrepublik war eine recht erfolgreiche Gesellschaft, was die wirtschaftlichen, wissenschaftlichen und politischen Einrichtungen und deren Lage betraf, während die Deutsche Demokratische Republik nicht nur politisch und wirtschaftlich, sondern auch hinsichtlich ihrer internationalen Anerkennung und Reputation, ihrer wissenschaftlichen Ansprüche und Kapazität in großen Schwierigkeiten steckte. Defizite als Folge mangelnder internationaler Kommunikation und verhinderter Gespräche stellten sich allenthalten heraus.

Diese weitgehend asymmetrische Situation, die sich aus vier Jahrzehnten von Misstrauen geprägter, oft feindseliger Beziehungen nach der gemeinsamen Katastrophe von 1945 ergeben hatte und in aktiver Beteiligung an den verschiedenen Perioden des Kalten Kriegs zum Ausdruck gekommen war, bildete den Hintergrund für die gegenseitigen Beurteilungen und Erwartungen im Prozess der Wiedervereinigung.

Was die verschiedenen Akteure in dieser Situation erwarteten, war eine entsprechende Assimilation der sehr unterschiedlichen Bedingungen - streng nach dem erfolgreichen westlichen Muster natürlich, dem mal mehr und mal weniger Vertrauen entgegengebracht wurde. Aber von Beginn an erwiesen sich die Probleme als ebenso groß wie die Erwartungen. Wie weit die faktischen Bedingungen von den gedachten Möglichkeiten einer sanften Wiedervereinigung abwichen, wurde dramatisch deutlich im Fall der Berliner Universität. Gegründet im Jahre 1810 nach dem berühmten Konzept der Einheit von Forschung und Lehre Wilhelm von Humboldts, lag sie im Osten der nach dem Zweiten Weltkrieg geteilten Stadt. 1948 hatte die Sezession einer aktiven Fraktion des Lehrpersonals und der Studenten zur Gründung der »Freien Universität « im amerikanischen Sektor von Berlin geführt, um dem sich ankündigenden ideologischen Druck in Lehre und Forschung zu entgehen. Eine Konsequenz daraus war, dass man 1949 die ursprüngliche »Friedrich-Wilhelms-Universität " in »Humboldt-Universität " umbenannte, wobei man sich auf beide Humboldt-Brüder bezog, auf Wilhelm und auf Alexander. Nach mehr als vier Jahrzehnten getrennten Wachsens und Entfaltens der zwei Berliner Universitäten in ihrer jeweiligen Umgebung war eine Wiedervereinigung der beiden Institutionen völlig undenkbar geworden und hätte heftigen Widerstand hervorgerufen. Es mussten also bei der weiteren Entwicklung Lösungen gefunden werden, die der tatsächlichen Lage gerecht wurden. Die zwei Universitäten mussten einfach miteinander auskommen. Inzwischen sind Humboldt- und Freie Universität aktive Teilnehmer eines gemeinsamen sogenannten Kompetenz-Clusters und eines ganzen Netzwerks von gemeinsamen Unternehmungen geworden.

\subsection{Die strategischen Vorschläge des Wissenschaftsrats}

Während die allgemeinen Erwartungen an die politische Vereinigung ziemlich offensichtlich waren, erwiesen sich die tatsächlichen Möglichkeiten und notwendigen Entscheidungen in den unterschiedlichen Bereichen als alles andere als einfach und klar. In einer informellen gemeinsamen Beratung unter der Federführung der Ostund Westminister für Forschung und Erziehung (das sogenannte »Kamingespräch «) 
debattierte man über die allgemeinen Ziele und die in Bezug auf Lehre und Forschung zu ergreifenden Maßnahmen. Das abschließende, aber noch recht vage Ziel wurde als eine vereinigte Landschaft der Wissenschaft in einem vereinigten Land beschrieben. Die Topoi der Debatte betrafen natürlich ausschließlich die Probleme der »neuen Länder", also die Situation der Wissenschaft in der DDR. Die Formulierung einer Grundperspektive, die Suche nach angemessenen Mitteln und konkreten Maßnahmen, die es zu implementieren gelte, betrafen ausschließlich Einrichtungen und einzurichtende Programme der östlichen Länder. Obwohl die Vereinigung eine Angelegenheit beider deutscher Staaten war, war die Veränderung westdeutscher Strukturen an keiner Stelle ein Thema.

In dieser Situation erwies es sich als bedeutender Vorteil, dass die Institutionenlandschaft der akademischen Selbstverwaltung in der Bundesrepublik eine Art strategischen Rat besaß, den »Wissenschaftsrat ", der sich darin bewährt hatte, Orientierung zu bieten und Vorschläge für politische Entscheidungen zu liefern, insofern sie Forschung und höhere Bildung betrafen. Die Mitglieder des Wissenschaftsrats waren erfahrene Gelehrte aller Disziplinen, ernannt vom Bundespräsidenten. Dieser Rat wurde beauftragt, Vorschläge zur Reorganisation der ostdeutschen Wissenschaftseinrichtungen zu machen und umzusetzen.

Das war einerseits ein hoffnungsloses Unterfangen: Der Wissenschaftsrat war nicht in der Lage, eine Blaupause für das komplette Forschungs- und Bildungssystem eines Landes zu entwickeln, obwohl das faktisch die gestellte Aufgabe war. Der reine Arbeitsaufwand für die Inspizierung, Evaluierung und die Anweisungen zur Neuorganisation für mehr als ein Dutzend Volluniversitäten und eine noch weit größere Zahl pädagogischer und anderer außeruniversitärer wissenschaftlicher Einrichtungen (darunter der 65 Akademie-Institute) übertraf die reguläre Kapazität des Wissenschaftsrats um Größenordnungen. Andererseits war dies die angemessenste Option, an die zu denken war, um das zu verwirklichen, was als Programm im »Kamingespräch " akzeptiert worden war. Tatsächlich war ein großer Teil der westdeutschen akademischen Gemeinschaft in der einen oder anderen Weise in diesen Prozess involviert.

Zur Lösung der Aufgabe bildete der Wissenschaftsrat eine Reihe von Kommissionen, die mit Fachleuten der verschiedenen Disziplinen besetzt wurden. Sie waren gehalten, alle relevanten ostdeutschen Einrichtungen zu inspizieren und auf der Basis ihrer lokalen und allgemeinen Eindrücke und Erfahrungen strategische Vorschläge für die weiter zu unternehmenden Schritte zu entwickeln.

Drei generelle Punkte sind hier anzumerken, die das mögliche Resultat dieser Bemühungen begrenzen mussten. Zunächst und ganz allgemein: Die Forderungen nach Vereinheitlichung und Reform der Universitäten betrafen die beiden Teile Deutschlands in ganz unterschiedlicher Weise. Vereinheitlichung wurde vornehmlich als Erfordernis zur Verbesserung der Situation im östlichen Teil betrachtet, während die Notwendigkeit zur Reform als ein aktuelles Desiderat in der Bundesrepublik deutlich geworden war gerade zu dem Zeitpunkt, als die politische Einigung alles veränderte.

Zum zweiten wurden der Wissenschaftsrat und seine Kommissionen gebeten, Vorschläge für strukturelle und substanzielle Entscheidungen zu entwickeln und zu 
legitimieren. Ihre tatsächliche Ausführung - die Entscheidung darüber, was dann wirklich geschah - war aber häufig eine Sache der Landesregierungen und der örtlichen Verwaltung. Es mussten also die sorgfältig ausgefeilten, wissenschaftlichen Überlegungen und Bewertungen mit ganz unterschiedlichen Arten praktischer und nicht wissenschaftlicher Bedingungen vermittelt werden.

Denn im Verfassungsrecht der Bundesrepublik gehören Wissenschaft und Bildung prinzipiell zum Rechtsbereich der Länder. Deshalb ist es in der Regel die Länderebene, nicht die Bundesebene, die entscheidet - und bei der Struktur der Fakultäten handelt es sich um einen einschlägigen Fall.

\subsection{Aspekte der Neuorganisierung}

Die Notwendigkeit zur Veränderung und Verbesserung der Situation auf der Grundlage der Empfehlungen des Wissenschaftsrats war umstritten und wurde nicht von allen Mitarbeitern und Angestellten der ostdeutschen Einrichtungen gleichermaßen akzeptiert, sondern je nach ihrer bisherigen Position und politischen Überzeugung verschieden bewertet. Aber unabhängig von unterschiedlichen Bewertungen und Überzeugungen gab es ein recht allgemeines Übereinkommen, dass einerseits ein großer Teil der ideologieneutralen Wissenschaft mit ihren im Durchschnitt sinnvollen Ergebnissen anerkannt wurde, während auf der anderen Seite zwei allgemeine Typen von Defiziten nicht infrage gestellt wurden.

Dabei handelte es sich zum einen um Einschränkungen und Defizite, die aus politischer Einmischung und ideologischer Indoktrination durch die Regierungspartei entstanden waren, die allen inhaltlichen und Richtungsfragen in Forschungsprogrammen und in wissenschaftlichen Erklärungen die Doktrin des sogenannten Marxismus-Leninismus übergestülpt hatte.

Zum zweiten gab es weniger spezifische Unzulänglichkeiten, die sich aus der wirtschaftlich schwierigen Situation der verschiedenen Einrichtungen ergaben, Mängel in der technischen Ausrüstung, Mängel in der Kommunikation wegen beschränkter Kontakte und die Behinderung der Teilnahme am internationalen Austausch.

Diese beiden Aspekte waren nicht unabhängig voneinander, aber sie hatten unterschiedliche Gründe und waren verantwortlich für ziemlich ungleichartige Wirkungen, und sie hatten ungleiche Bedeutung in den verschiedenen Disziplinen. Ideologische Beschränkungen hatten insbesondere die Sozial- und Geisteswissenschaften behindert, in denen die Partei versuchte, autokratisch die Agenda zu bestimmen. Die Naturwissenschaften und die technologischen Fächer hatten andererseits mehr an faktischer Knappheit, an materiellen Einschränkungen und Informationsdefiziten, an unzureichendem materiellen Nachschub und fehlender technischer Innovation gelitten.

Zwar waren die »Gesellschaftswissenschaften « viel stärker von der offiziellen, parteimäßigen Version des Marxismus-Leninismus vergiftet als die Naturwissenschaften, aber die Grenzen waren nicht einfach und klar zugeschnitten, sondern verschwammen unter manchmal sehr merkwürdigen Bedingungen, die von den lokalen Behörden, persönlichen Präferenzen oder individueller Dummheit aufgezwungen wurden. Ein exemplarischer Fall ist Geschichte der Kybernetik in der 
DDR, die zuerst diffamiert, dann wissenschaftlich anerkannt und schließlich erneut als Pseudowissenschaft abgestempelt wurde.

Im Hinblick auf diese geschilderten allgemeinen Bedingungen und auf der Grundlage detaillierter örtlicher Inspektionen und sorgfältig diskutierter Orientierungslinien brachten der Wissenschaftsrat und seine Verbündeten bis ins Einzelne gehende Vorschläge und Leitfäden heraus, die es den akademischen Gremien erlaubten, ihrer jeweiligen Zuständigkeit entsprechend vorzugehen. Die Vorschläge umfassten auch Richtlinien und Prinzipien, nach denen die Organisations- und Personalstruktur der reorganisierten Fakultäten entwickelt und die Gründungskommissionen zusammengesetzt werden sollten.

\subsection{Die große Veränderung}

Die Beratungen und Debatten, die die Entwicklungen vorbereiteten und begleiteten, waren stets eine Angelegenheit ausgewogener Teilnahme von Ost- und Westkollegen, wenn auch aus offensichtlichen Gründen in ungleicher Proportion, mit einer natürlichen Dominanz des westlichen Wissenschaftsestablishments. Das war die genauso natürliche wie unvermeidliche Konsequenz der zuvor beschriebenen Asymmetrie, und es hatte seine ebenso natürlichen Folgen in allen Phasen der Umsetzung. Die Schaffung einer »vereinigten Wissenschaftslandschaft « hatte recht unterschiedliche Auswirkungen für die Universitäten und außeruniversitären Institutionen im östlichen Landesteil. Das ist im Folgenden zu erläutern.

\section{Reorganisation der Universitäten}

Trotz aller Verwerfungen war der grundlegende Begriff der Universität in Ost und West immer noch der gleiche. Von daher bestand die hauptsächliche Ausrichtung der Vereinigung darin, die Unterschiede und Proportionsverschiebungen in den östlichen Universitäten zu überwinden. Zu diesem Zweck wurde gewöhnlich eine Kernkommission benannt, die das bisherige Personal zu evaluieren, eine angepasste institutionelle Struktur zu formulieren und eine neue Fakultät zu berufen hatte. Hier ist ein konkretes Beispiel für die völlig außergewöhnliche dieser Kommissionen.

Die »Berufungskommission ", die für drei philologische Abteilungen an der Humboldt-Universität zuständig war, hatte neun Mitglieder, darunter drei, die nicht zur Professorenebene gehörten, drei Kollegen von der Humboldt-Universität und drei externe Kollegen aus dem Westen. Leiter der Kommission war (wie üblich) ein Kollege aus dem Westen mit hoher Reputation und großer Erfahrung in der akademischen Selbstverwaltung. Diese sechs professoralen Kollegen mussten die Bewerbungen für 36 Professuren prüfen, auswerten und klassifizieren, um innerhalb von zwei Jahren 36 Vorschläge vorzulegen. Unter den Bewerbern waren zwar auch bisherige Mitglieder der Humboldt-Universität, aber eine viel größere Zahl externer Kandidaten. Das abschließende Ergebnis zu beurteilen ist nicht leicht, da die vorgeschlagenen Listen von der Verwaltung entschieden wurden und die Regierung in manchen Fällen die Liste der verfügbaren Positionen anlassbedingt verändert hatte. 
Grob geschätzt, kamen von den schließlich ernannten Wissenschaftlern in etwa ein Drittel aus dem Osten und zwei Drittel aus dem Westen. Das machte die Abteilungen durch neue Kompetenzen und eine andere Ausrichtung attraktiver, aber es bedeutete auch, dass nicht alle früheren Kollegen ihre bisherige Position behalten konnten.

Das erläuterte Beispiel ist charakteristisch, aber natürlich finden sich zahlreiche und zum Teil erhebliche Abwandlungen in anderen Fällen, die aus den mitunter drastisch verschiedenen, nicht nur fachspezifischen Gegebenheiten entstehen.

Ein schwerwiegendes Problem muss bei dieser Illustration der Neuernennung einer ganzen Fakultät noch erwähnt werden. In der Regel hatten die ostdeutschen Universitäten eine Struktur von Lehrstühlen und Assistenten, die markant von den westdeutschen Standards abwich. Charakteristischerweise gab es neben dem Personal in den Sekretariaten eine ziemlich große Gruppe akademischer Beschäftigter, die Mittelbau genannt wurde, darunter Lektoren, Forschungs- und Lehrassistenten, die zu integrieren das westliche System nicht bereit war und für die es nicht einmal eine Bezeichnung hatte. Obwohl dieser Mittelbau sehr wichtige Funktionen für die Lehre und Forschung hatte, musste ein großer Teil dieses Personals unterhalb der Stufe der Professoren ausgeschlossen werden, was nicht nur zu Problemen bei der Lösung bestimmter praktischer Aufgaben führte, sondern auch zu Schwierigkeiten für nicht wenige bisher normale Karrieren, für die nur schwer eine Lösung zu finden war.

\section{Die Integration der außeruniversitären Forschung}

Die Lage der außeruniversitären Forschungseinrichtungen war sehr anders - vor allem, aber keineswegs nur für die Berliner Akademie der Wissenschaften. Diese Organisation mit ihren 65 Forschungseinheiten hatte in etwa die Größe der MaxPlanck-Gesellschaft. Es war von Anfang an klar, dass die »vereinigte Wissenschaftslandschaft " keine zweite, ebenso große, auf Grundlagenforschung spezialisierte Organisation aufnehmen und eingliedern würde.

Von daher waren die Pläne auf Seiten der DDR-Akademie, ihre Einrichtungen in eine Leibniz-Gesellschaft für Grundlagenforschung zu überführen, zum Scheitern verurteilt. Die Akademie musste auf das Format der traditionellen Gelehrten-Gesellschaft zurückgeführt werden, nach dem Vorbild der in der sogenannten Union der Akademien zusammengefassten westdeutschen Traditionsunternehmen. In diesem Zusammenschluss nimmt sie - übrigens zusammen mit der Sächsischen Akademie der Wissenschaften - an langfristigen Akademieprogrammen in den Humanwissenschaften teil, ein wirksam unterstützter und erfolgreich verwirklichter Vorschlag der Neugestaltung. Als Folge davon mussten fast alle Forschungsinstitute, die von sehr unterschiedlicher Art, Größe und Qualität waren, entweder aufgelöst oder in andere, bereits existierende Einheiten eingepasst werden. Um im Rahmen dieser Konditionen Lösungen für die Projekte und Abteilungen (vor allem der Naturwissenschaften) zu entwickeln, die im Wesentlichen den üblichen methodologischen und theoretischen Standards genügten, wurde eine beachtliche Mischung verschiedener Optionen aufgeboten. Diese Versuche konvergierten in gewissem Maße mit 
Entwicklungen im Bereich der außeruniversitären Forschung der Bundesrepublik, wo in den 1980er Jahren eine Reihe von völlig unterschiedlichen Gelegenheiten und Bedürfnissen zu einer Anzahl von sogenannten »Großforschungszentren « geführt hatte, von denen 17 im Jahr 1995 in der Helmholtz-Gemeinschaft deutscher Forschungszentren zusammengeführt wurden. Ebenfalls 1995 verband man aufgrund einer wieder anderen, in mancher Hinsicht vergleichbaren Entwicklung 80 Einheiten für Grundlagenforschung, die zu verschiedenen Gelegenheiten mit besonderen Bestimmungen gegründet worden waren, zu fünf Sektionen, die zusammen die Leibniz-Gemeinschaft bildeten (nicht zu verwechseln, wenn auch implizit gewissermaßen überlappend, mit der Leibniz-Gesellschaft, wie sie von der DDR-Akademie in ihrer Endphase skizziert worden war), wobei nun etwa 30 dieser Einrichtungen in modifizierter Form an Projekten und Themen von Akademie-Instituten weiterarbeiten konnten.

Es war gewiss keine zufällige Koinzidenz, dass in dieser Periode zusätzlich zur Max-Planck- und zur Fraunhofer-Gesellschaft mit der Helmholtz- und der LeibnizGemeinschaft zwei weitere Forschungsorganisationen fest etabliert wurden, in denen ein gewisser Teil der ernstzunehmenden Akademie-Forschung als neue Institute integriert wurde. Die Einzelheiten dieser verschiedenen Fälle können hier nicht ausbuchstabiert werden. Aber es verdient sicher vermerkt zu werden, dass auf diese Weise überlebende Teile der DDR-Akademie indirekt und in substanzieller Modifikation Teil der außeruniversitären Forschungslandschaft Gesamtdeutschlands geworden sind.

Diese Entwicklungen verlangten beträchtliche Anstrengungen auf beiden Seiten, aber sie kamen nach objektiven Standards recht erfolgreich voran, soweit es die naturwissenschaftlichen und technischen Einrichtungen betraf. Im Bereich der Geistes- und Sozialwissenschaften, wo es ideologische Vorurteile zu neutralisieren galt, lagen die Dinge komplizierter. Schließlich aber wurde in diesem Bereich unter der Ägide der Max-Planck-Gesellschaft eine echte Neuerung erfolgreich: Nach mehreren Jahren mit Interventionen und Schwierigkeiten wurden sieben » Geisteswissenschaftliche Zentren " eingerichtet und als neuer Typus einer Forschungseinrichtung außerhalb der Universitäten anerkannt.

Durch die Trennung der Akademie von ihren Forschungsinstituten verloren 24.000 der Beschäftigten dieser Einrichtung unvermeidlich ihre Stelle. In dem Ausmaß, in dem ihre Projekte in die Helmholtz- oder Leibniz-Institute oder in die Geisteswissenschaftlichen Zentren integriert wurden oder als Langfrist-Projekte der Akademie, die als Gelehrtengesellschaft wiederbegründet worden war, weiterbearbeitet wurden, war die Fortsetzung der Beschäftigung und der Forschung garantiert. Es war allerdings von Anfang an klar, dass davon bestenfalls die Hälfte der Beschäftigten profitieren konnte. In Erwartung der Probleme, die aus dieser Situation entstanden, schlug der Wissenschaftsrat ein Integrationsprogramm vor, das aus drei Teilen bestand. Erstens wurden alle Beschäftigten gleichermaßen nach ihren Fähigkeiten bewertet. Zweitens wurde für jene, die trotz ihrer positiven Bewertung nicht in eines der neuen Programme eingebunden worden waren, Empfehlungen für zunächst befristete Stellen ausgegeben, wodurch die Personalausstattung an den Universitäten verbessert werden sollte. Drittens wurden diese Positionen als dauer- 
haft eingeplant, da man sich darüber klar war, dass auch weiterhin qualifiziertes Personal benötigt werden würde.

Waren der erste und der zweite Schritt dieses Integrationsprogramms ziemlich erfolgreich, so scheiterte der dritte Teil des Programms völlig. Das war freilich nicht überraschend, wenn man an die oben erwähnten Probleme mit dem Mittelbau denkt. Dieser Einbruch war der dramatischste Misserfolg aus der Liste der Vorschläge, die vom Wissenschaftsrat ausgegangen waren. Versuche, die Folgen dieses Scheiterns nachträglich aufzufangen, wirkten eher hilflos.

\section{DFG: Ein neues Verständnis von Forschung}

Eine entscheidende Komponente für die vereinigte Wissenschaftslandschaft war die Einbeziehung Ostdeutschlands in die Verantwortung der Deutschen Forschungsgemeinschaft wie auch der anderen Stiftungen und Einrichtungen der Wissenschaftsförderung. Diese gewissermaßen automatische Konsequenz der Vereinigung war von unvergleichlicher Bedeutung für alle Aspekte der Forschung im östlichen Teil. Obwohl das Budget der DFG entsprechend ausgeweitet werden musste, blieb die Organisationsstruktur praktisch unverändert. Die Möglichkeit des Zugangs zu all ihren Mitteln eröffnete für die ostdeutschen Universitäten eine völlig neue, bislang unbekannte Dimension für Forschung und wissenschaftliche Aktivitäten. Die tatsächliche Beteiligung an der Wahl und den Verantwortlichkeiten des Beirats, der Beratungs- und Beurteilungskomitees sowie an der gesamten Bandbreite der Selbstverwaltung machte aus dem formalen Begriff der vereinigten Wissenschaftslandschaft eine praktische Erfahrung mit all ihren Vorzügen und Pferdefüßen.

\section{Einige Folgen}

\subsection{Ungleicher Transfer}

Die Asymmetrie, die zu Beginn beschrieben wurde, war mit allen ihren Konsequenzen in Bezug auf praktisch alle Gesichtspunkte der Entwicklung und der Ergebnisse, die zur Debatte gestanden hatten, zu spüren - und zwar für einen längeren Zeitraum, als man ursprünglich erwartet hatte.

\section{West-Ost-Asymmetrie}

Vor der Vereinigung gab es eine starke Asymmetrie der Interessenrichtung, bei der es überwiegend um die Aufmerksamkeit des Ostens für den Westen ging, während das Interesse des Westens am Osten begrenzt war. Aus leicht einsichtigen Gründen verlief die tatsächliche Integration nach der Vereinigung in umgekehrter Richtung: Die strategischen Entscheidungen und organisatorischen Folgen betrafen ausschließlich Einrichtungen und Programme im Osten; außerdem war offensichtlich, dass die Protagonisten der anforderungsreichen Aktivitäten hauptsächlich erfahrene 
Kolleginnen und Kollegen aus dem Westen waren, während die Ostkollegen lediglich als Berater und örtliche Spezialisten teilnahmen. Und es muss hinzugefügt werden: Dieses Engagement war von enormer Bedeutung für die östlichen Einrichtungen, durch das eine Reihe von andernfalls vorhersehbaren Kalamitäten vermieden wurde.

Als gewissermaßen natürliche Folge dieser Umstände fand ein ungleicher Transfer von Personal statt. Im Zuge der Evaluierung und Reorganisation eines großen Teils der Positionen und Abteilungen lud man gezielt zu Bewerbungen ein, die dadurch, dass neue, externe Berufungen ausgeschrieben wurden, überwiegend zur Einstellung von Westkollegen führte. Das sicherte vielen Abteilungen eine engagierte Atmosphäre, starke Motivation und höchst willkommene Erfahrung, da zu einem großen Teil hochqualifizierte und ernsthaft interessierte Bewerber eingestellt wurden. Es wäre aber nicht korrekt, die Tatsache zu übersehen, dass auch eine große Zahl von zweitrangigen Kandidaten eine Chance bekam, die sie nicht bekommen hätten ohne diese ungewöhnliche Art der Neubesetzung von Positionen. Insgesamt war die Richtung des Transfers recht einheitlich, die Qualität nicht immer nur erstklassig und das Ergebnis eine auf jeden Fall bemerkenswerte neue Periode.

Wiederum aus einsichtigen Gründen war ein ausgleichender Transfer von ostdeutschen Bewerbern auf westdeutsche Positionen nicht wirklich ein Thema: Für eine entsprechende Reorganisation im Westen sah man keine aktuellen Anlässe. Auf diese Weise wurde das naturgemäße Ausbleiben eines korrespondierenden OstWest-Transfers zu einem der Gründe, warum das oben beschriebene Integrationsprogramm für Forscher der Akademien zum Scheitern verurteilt war.

\section{Spätere Bilanz}

In dem Maße, in dem die Vereinigung langsam zur Alltagsrealität wurde, wurden auch die Asymmetrien, die hier aufzuzählen waren, überwunden. Tatsächlich machte die stufenweise Normalisierung der Beziehungen zwischen den unterschiedlichen Landesteilen und ihren Forschungseinrichtungen Veränderungen in beiden Richtungen zu einem ganz natürlichen Vorgang, auch was Verschiebungen von west- zu ostdeutschen Einrichtungen betraf. Es versteht sich von selbst, dass diese Veränderungen vollständig verschieden sind von jenen aus der anfänglichen Vereinigungsphase, da sie nun auf einer normalen akademischen Motivation beruhen und Leute betreffen, die größtenteils die Ost-West-Sozialisation hinter sich gelassen haben. Von daher ist der Ost-West-Transfer Teil der Normalisierung nach der mehr oder weniger erfolgreichen Vereinigung der Wissenschaftslandschaft.

\subsection{Die versäumte Reform}

Wie oben beschrieben, hätte der völlig außergewöhnliche Zustand einer zusammengebrochenen Verwaltung als Chance wahrgenommen werden können für einen radikalen Neubeginn. Aber immer da, wo die Vereinigung Prozesse der Reform und der Innovation initiierte, wurde das als Angelegenheit der ostdeutschen Einrichtun- 
gen verstanden, wobei man sich auf weitgehend erfolgreiche, aber gewöhnlich konservative Prinzipien der westlichen Einrichtungen verließ und damit natürlich alle Überlegungen zu erwünschten Reformen im Westen blockierte. In der Folge gab es Evaluationen der Beschäftigten und der Konzepte in allen östlichen Einrichtungen, aber obwohl vergleichbare Maßnahmen ganz generell als notwendig erkannt wurden, war an entsprechende Schritte in den alten Bundesländern in der Zeit der Reorganisation der ostdeutschen Länder nicht zu denken - sie wurden einfach als nicht realisierbar betrachtet.

Es brauchte mehr als ein Jahrzehnt und mehrere internationale Vergleiche, bis völlig unabhängig von der Vereinigung neue Bemühungen entstanden, die Universitäten der Länder miteinander zu vergleichen und neue »Exzellenz «-Initiativen einzurichten, die nun die ostdeutschen Universitäten völlig selbstverständlich einbezogen.

\subsection{Anpassungen, Schnitte, Normalisierung}

Die Integration und Vereinigung im Allgemeinen und der wissenschaftlichen Einrichtungen im Besonderen war ein längerer und komplizierterer Prozess als ursprünglich erwartet, und nicht alle Vorschläge und Empfehlungen, die es dazu gab, wurden wie geplant umgesetzt. Großzügige Lösungen, die zu Anfang versprochen worden waren, mussten zurückgestutzt werden, Alltagsbelange, die nicht immer nur irrational waren, sorgten für Einschnitte und Anpassungen, denn die Maßstäbe der Verwaltung waren nicht immer dieselben wie diejenigen der Wissenschaftsgemeinschaft. Nicht nur die Opfer der nicht erfolgreichen Programme für die Integration, sondern auch die Vertreter von nicht verwirklichten Ideen für neue Forschungsprogramme blieben unbefriedigt. Im Großen und Ganzen aber war die Vereinigung so erfolgreich, wie man erwarten konnte, und sie schuf tatsächlich so etwas wie eine vereinigte Wissenschaftslandschaft. Wie schon in Bezug auf die beiden Berliner Universitäten erwähnt, entwickelten sich inzwischen eine produktive Kooperation und eine Fülle von gemeinsamen Unternehmungen.

In manchen Fällen überlebten eigenartige Reste aus verwaltungstechnischen Gründen, so etwa, wenn - wie bis vor kurzem - Leute im selben Raum sitzend dieselbe Arbeit machten, aber für ein unterschiedliches Gehalt, nur weil sie vor zwei Jahrzehnten Bürger zweier verschiedener Staaten waren. Doch das sind seltsame Ausnahmen, nicht wirkliche Probleme.

Im Allgemeinen aber gilt, dass so, wie es beim Spaziergang durch Berlin jetzt schwierig ist, zu erkennen, wo ehemals die Berliner Mauer stand, auch ein Unterschied zwischen früherer ostdeutscher und westdeutscher Wissenschaft schwer auszumachen ist. 
Zusammenfassung: Die Wiedervereinigung bedeutete für die Wissenschaft eine schwere Aufgabe, deren sich insbesondere der Wissenschaftsrat annahm. Dabei wurden orientierende Vorstellungen entwickelt, aber auch Erwartungen geweckt, von denen manche später nicht erfüllt wurden, sodass die mitunter drastischen Änderungen neben vielen erfolgreichen Entwicklungen auch zu ernsten Enttäuschungen führten. Insgesamt war die Vereinigung nicht erfolglos.

Stichworte: Wissenschaftslandschaft, Wiedervereinigung, Wissenschaftsrat, Evaluierung, Wissenschaftler-Integrationsprogramm (WIP)

\section{The illusion of a new beginning. Science and reunification}

Summary: Reunification brought about the very difficult task to integrate two quite differently developed systems of research and education. This task was primarily adopted by the Science Council, which developed orienting proposals and helpful expectations, some of which were not fulfilled, however. Thus besides rather successful developments, the sometimes harsh changes led occasionally to serious disappointments. Altogether, though, the unification was not without success.

Keywords: landscape of science, reunification, Wissenschaftsrat, German Council of Science and Humanities, evaluation, program of integration of scientists (WIP)

\section{Autor}

Prof. Dr. Manfred Bierwisch

Mitglied der Berlin Brandenburgischen Akademie der Wissenschaften

Rüdesheimer Str. 6

14197 Berlin

bierwisch@gmx.net 